\title{
The relationship between sport-specific training and a perceptuo-motor skills assessment as part of talent identification in young table tennis players (8-10 years)
}

\author{
Irene Faber ${ }^{1,2}$, Maja Zamoscinska', Jan Willem Teunissen ${ }^{3,4}$, Johan Pion ${ }^{3,4}$ \\ 'Institute of Sport Science, University of Oldenburg, Germany \\ ${ }^{2}$ International Table Tennis Federation, Switzerland \\ ${ }^{3}$ Institute for Studies in Sports and Exercise, HAN University of Applied Sciences, The \\ Netherlands \\ ${ }^{4}$ Faculty of Medicine and Health Sciences, Ghent University, Ghent, Belgium
}

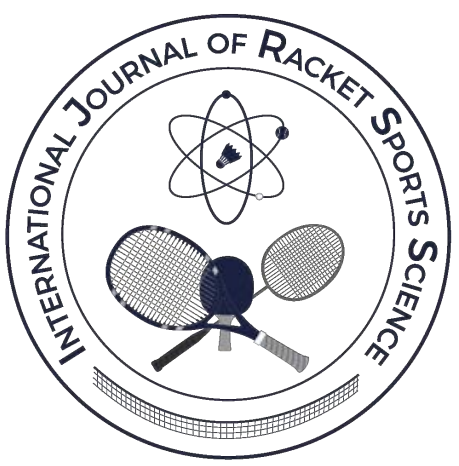

\begin{abstract}
A perceptuo-motor skills assessment was developed to better estimate the potential of youth table tennis players (8-10 years). Sport-specific training experience might influence the outcomes of this assessment and hinder a fair interpretation. Consequently, the aim of this study was to evaluate the relationship between the training experience (i.e. quantity and quality) and the perceptuo-motor skills assessment outcomes in youth table tennis players. For this purpose, data were collected during the national association's talent days in the Netherlands (2010-2015). In total, 372 young table tennis players (8-10 years) were assessed by a perceptuo-motor skills questionnaire covering current training (hours/week), total training volume (hours) and quality of the trainer (high versus low). Non-parametric partial correlation analyses showed that training quantity outcomes (i.e. current training and training volume) are significantly associated with the test items of speed while dribbling, aiming at target and eye-hand coordination in both sexes with small to medium effect sizes. The multivariate GLM analyses revealed no significant differences between players receiving high versus low quality training regarding the perceptuo-motor skills assessment. The results indicated only a small transfer of skill and a substantial amount of task specificity; as such it seems legitimate to use the perceptuo-motor skills assessment as part of a talent identification programme. However, it seems sensible to take the training volume into account for a fair interpretation of the estimation of potential, especially when large differences exist between players regarding the training experience. Future studies using a longitudinal design could reveal more about the effect of training.
\end{abstract}

Keywords: Task/Learning Specificity, Skill Transfer, Talent Identification, Aptitude, Child, Racket Sports

Correspondence author: Irene Faber

E-mail: irene.faber@uni-oldenburg.de

Cite this article as:

Faber, I., Zamoscinska, M., Teunissen, J. W., \& Pion, J. (2020). The relationship between sport-specific training and a perceptuo-motor skills assessment as part of talent identification in young table tennis players (8-10 years). International Journal of Racket Sports Science, 2(1), 42-54. 


\section{Introduction}

Talent identification and development are important pillars of success of national sports associations nowadays (de Bosscher, Bingham, Shibli, Bottenburg, \& Knop, 2007). Consequently, talent programs have gained priority in many sports during the last decades, including large investments regarding the search and guidance of young talented players who strive to reach world's highest level (e.g. Elferink-Gemser, 2005; Huijgen, 2013; Pion, 2015; Kramer, 2019; Stoter, 2020). This is also the case in the table tennis sport (Faber, 2016). It appears that national table tennis associations have put special efforts specifically into talent identification as part of the talent programs to increase their success-rate. This is likely due to the facts that table tennis is generally considered as an early starting sport and players have a relatively high age of peak performance especially in West-Europe (Faber, Damsma, Pion, in press). Early recognition of talented players at a young age (7-12 years) is anticipated to increase efficiency and effectiveness of the intensive training programs by including only youth players with the highest potential. Therefore, accurate talent identification is expected to be a part of a costeffective solution to keep up with the global medal race, particularly for relatively small countries with scarce (financial) resources (de Bosscher et al., 2007; Faber, 2016; Pion, Hohmann, Liu, Lenoir, \& Segers, 2017).

One initiative following this strategy was conducted by the Netherlands Table Tennis Association (NTTA). From 1998 to 2016, the NTTA developed and evaluated a perceptuo-motor skills assessment in cooperation with a team of sports scientists in order to better estimate the potential of young players ( $<11$ years) regarding the perceptuomotor domain. The current NTTA's perceptuo-motor skills assessment consists of eight test items (Table 1). These items are intentionally combined together to measure foundational abilities for table tennis (e.g. coordination, dynamic balance, accuracy, agility, ball control), which are crucial for developing excellent technical features (Faber, Elferink-Gemser,
Oosterveld, Twisk, J. W. \& Nijhuis-Van der Sanden, 2016). Evaluative studies show that the inter-rater reproducibility per test items is good (Intraclass Correlation Coefficient .73-.91 ( $\mathrm{p}<.01)$, Coefficient of Variation 1-15\%) (Faber, Elferink-Gemser, Oosterveld, \& Nijhuis-Van Der Sanden, 2014a; Faber, Nijhuis-Van Der Sanden, Elferink-Gemser, \& Oosterveld, 2015) and the test items are able to discriminate between performance levels (Faber et al., 2014a; Faber, Oosterveld, \& Nijhuis-Van der Sanden, 2014b; Faber, Pion, Munivrana, Faber, \& Nijhuis-Van der Sanden, 2018; Platvoet et al., 2018). Furthermore, an observational study shows promising results regarding the predictive value in a small sample over a period of 2.5 years. The assessment's test items significantly add to the prediction of future performance and explained $51 \%$ of the variance within the best-fitting model (Faber et al., 2016). As such, the NTTA has included this assessment as an instrument to support the identification of talented players in their talent pool at the annual national talent day beginning in 2010 .

As an extension to the aforementioned studies, it is important to address the association between the table tennis training and the assessment's outcomes in the current study. This relationship has not been taken into account so far, although the exposure to training is suggested as a possible confounder when interpreting test results for the estimation of potential (Elferink-Gemser, Jordet, Coelho E Silva, \& Visscher, 2011; Faber et al., 2015; Faber et al., 2016). If youth table tennis players would benefit from their sport-specific training experience (i.e. youth players with more and/or higher quality table tennis training experience gain better results on the assessment), this might prevent a fair estimation of potential. For that reason, authentic table tennis tasks were deliberately avoided to minimize or even eliminate the effect of training during the developing of the test items. The idea was that skills, which are not trained, are more appropriate to measure future potential than specific sport skills themselves (Faber et al., 2015; Gagné, 2004; Morrow, Jackson, Disch, \& Mood, 2011; Vaeyens, Lenoir, Williams, \& Philippaerts, 2008; Vandorpe et al., 2012). Indeed, 
task/learning specificity theories imply that motor abilities are specific to a particular task and that transfer among skills is quite low (de Camargo Barros, Tani, \& Corrêa, 2017; Ellison, Kearney, Sparks, Murphy, \& Marchant, 2018; Schmidt \& Lee, 2011; Sigmundsson, Trana, Polman, \& Haga, 2017).

On the other hand, previous studies on skill transfer show that a transfer is possible based on an underlying general motor ability and training can have an effect on trained and untrained skills (Behringer, vom Heede, Matthews, \& Mester, 2011; Farhat et al., 2016; Rylander, Karlsteen, Kougioumtzis, \& Gustafsson, 2019). In sports, this perhaps becomes most obvious when taking into account the evidence of successful transfers between sports on the elite level (Collins, Collins, MacNamara, \& Jones, 2014). Athletes seem to build upon previous skills and need considerably less hours to get to the elite level in their second target sport (Rea \& Lavallee 2015). For example, Rebecca Romero gained a silver medal in rowing in the quadruple sculls at the Olympics of 2004, transferred to cycling and won a gold medal in the individual pursuit at the Olympics of 2008. Based on Thorndike's theory (1914), it is proposed that 'identical elements' between tasks or the 'underlying processes' are transferable (Côté, Baker, \&
Abernethy, 2007; Schmidt \& Lee, 2011). As a nuance to this, Issurin (2013) stated that specificity of motor learning or the degree of transfer are affected by many variables. The amount of practice is considered to be the most important one, followed by structure of the practice and then specificity of learning (Ericsson, Krampe, \& Tesch-Romer, 1993; Schmidt \& Lee, 2011). Moreover, one especially needs to take training experience into account given the fact that less experienced athletes show greater sensitivity to any kind of training transfer than the more experienced ones (Gamble, 2006; Issurin, 2013).

Thus it appears that the current scientific evidence is ambiguous and prohibits a clear conclusion from studies with regard to, on the one hand 'task/learning specificity' and on the other hand 'transfer of skill', which seem contradictory. Consequently, it is proposed that empirical evidence of the relationship between the table tennis training and the assessment's outcomes is needed to have a better insight in the possible confounding effect of training on the estimation of table tennis potential. Accordingly, this study focuses on the following research question: Are the perceptuo-motor skills assessment outcomes related to the quantity and/or quality of the sport-specific training in youth table tennis players (8-10 years)? 
Table 1.

Current test items of the motor skills assessment of the Netherlands Table Tennis Association (Faber et al., 2016)

\begin{tabular}{|c|c|c|}
\hline Test item & Assessing: & Task: \\
\hline Sprint (s) & $\begin{array}{l}\text { The ability for quick accelerations and } \\
\text { turns in combination with a manual } \\
\text { task while picking up balls in a pyramid } \\
\text { shaped circuit. }\end{array}$ & $\begin{array}{l}\text { Gather and return five table tennis balls one by one } \\
\text { in a pyramid-shape circuit as fast as possible. Time } \\
\text { is measured in seconds. }\end{array}$ \\
\hline Agility (s) & $\begin{array}{l}\text { The ability to quickly coordinate gross } \\
\text { arm and leg movements simultaneously } \\
\text { while getting over a gymnastic cabinet } \\
\text { and under hurdles several times. }\end{array}$ & $\begin{array}{l}\text { Get through a circuit as fast as possible including } \\
\text { climbing over a gymnastics' cabinet (five times) } \\
\text { and under and over a low hurdle (four times). Time } \\
\text { is measured in seconds. }\end{array}$ \\
\hline $\begin{array}{l}\text { Vertical jump } \\
(\mathrm{cm})\end{array}$ & The ability to jump as high as possible. & $\begin{array}{l}\text { Stand next to a wall and jump and touch the wall } \\
\text { with your fingertips as high as possible. The } \\
\text { difference between the jumping height and } \\
\text { standing height with one arm up along the wall is } \\
\text { measured in centimetres. }\end{array}$ \\
\hline $\begin{array}{l}\text { Speed while } \\
\text { dribbling (s) }\end{array}$ & $\begin{array}{l}\text { The ability to control a basketball } \\
\text { during a dynamic task: sideward } \\
\text { zigzagging. }\end{array}$ & $\begin{array}{l}\text { Move sideways through a zig-zag circuit as fast as } \\
\text { possible while dribbling with a basketball using one } \\
\text { hand. Time is measured in seconds. }\end{array}$ \\
\hline $\begin{array}{l}\text { Aiming at } \\
\text { target } \\
\text { (points) }\end{array}$ & $\begin{array}{l}\text { The ability to hit a target at } 2.5 \mathrm{~m} \\
\text { distance precisely with a ball using a bat } \\
\text { while alternately using a forehand and } \\
\text { backhand stroke. }\end{array}$ & $\begin{array}{l}\text { Hit a round target }(\varnothing 60 \mathrm{~cm}) \text { on the floor at } 2.5 \text { - } \\
\text { meter distance with a table tennis ball using a } \\
\text { standard bat. Forehand and backhand need to be } \\
\text { used alternately during the attempts. A hit in the } \\
\text { target' centre ( } \varnothing 0.20 \mathrm{~m}) \text { or the outer ring yielded } 6 \\
\text { and } 4 \text { points, respectively. The total score of ten } \\
\text { attempts is registered as the final score. }\end{array}$ \\
\hline $\begin{array}{l}\text { Ball skills } \\
\text { (points) }\end{array}$ & $\begin{array}{l}\text { The ability to hit a target precisely by } \\
\text { throwing a table tennis ball via the } \\
\text { vertical positioned table tennis table } \\
\text { alternately from two distances. }\end{array}$ & $\begin{array}{l}\text { Hit a round target on the floor }(\varnothing 75 \mathrm{~cm}) \text { by } \\
\text { throwing a table tennis ball via a vertical table } \\
\text { tennis table from two different positions ( } 1 \text { - and } 2 \text { - } \\
\text { meter distance away from the target). A hit in the } \\
\text { centre }(\varnothing 0.335 \mathrm{~m}) \text { or the outer ring of the target } \\
\text { yielded } 2 \text { and } 1 \text { points, respectively. The total score } \\
\text { of the twenty attempts is registered as the final } \\
\text { score. }\end{array}$ \\
\hline $\begin{array}{l}\text { Throwing a } \\
\text { ball (m) }\end{array}$ & $\begin{array}{l}\text { The ability to combine the coordination } \\
\text { of an arm movement with high arm } \\
\text { velocity by throwing a table tennis ball } \\
\text { as far as possible. }\end{array}$ & $\begin{array}{l}\text { Throw a table tennis ball as far away as possible } \\
\text { with the preferred hand. The distance from the } \\
\text { starting point at the marked line to the point of the } \\
\text { ball's first bounce is measured in meters with one } \\
\text { decimal. }\end{array}$ \\
\hline $\begin{array}{l}\text { Eye-hand } \\
\text { coordination } \\
\text { (points) }\end{array}$ & $\begin{array}{l}\text { The ability to make accurate and } \\
\text { cooperative hand and arm movements } \\
\text { at a high rate by throwing a table tennis } \\
\text { ball towards a vertically positioned table } \\
\text { tennis table with one hand and catching } \\
\text { is with the other hand and repeating } \\
\text { this alternately. }\end{array}$ & $\begin{array}{l}\text { Throw a ball at a vertical table tennis table at } 1 \text { - } \\
\text { meter distance with one hand and to catch the ball } \\
\text { correctly with the other hand as frequently as } \\
\text { possible in } 30 \text { seconds. The number of correct } \\
\text { catches is scored. }\end{array}$ \\
\hline
\end{tabular}




\section{Materials and methods}

\section{Study design and ethical statement}

This study used a cross-sectional design including a data-collection carried out at the National Training Center Papendal in the Netherlands during six annual NTTA's national talent days (2010-2015). This study and its informed consent procedure were approved by the ethical committee of the Medical Spectrum Twente (Medical School Twente, Institute for Applied Sciences, Enschede, the Netherlands; MTC/11069.oos 18-2-2011) in full compliance with the declaration of Helsinki. At least 1 month prior to the measurements, players and their parents were informed in writing by the NTTA that data, sampled during the NTTA's national talent day, would be anonymously collected for this study. There was full opportunity for players and their parents to ask for more information concerning this study and/or refuse provision of the anonymous results. All data were recorded in an anonymous data set by the NTTA; the ethical committee consulted waived the need of a written parental and player's informed consent.

\section{Participants}

Young table tennis players were recruited during the annual national talent days of 2010 to 2015. Regional technical staff from each department $(n=8$; Holland-Noord, Zuid-West, West, Noord, Limburg, Gelre, Midden and Oost) were instructed to select youth members of their department with the highest potential for national elite table tennis regarding both physical and mental aspects. A maximum of 8 boys and 8 girls were selected per department. Inclusion criteria were: an age between 8 and 10 years and being a member of a table tennis club associated with the NTTA. Players with injuries were excluded from the study.

\section{Data collection}

\section{Perceptuo-motor skills assessment}

The perceptuo-motor skills assessment of the young players consists of eight test items (Table 1). The standardization of all test items is captured in protocols, which includes a detailed description of materials, set-up, assignment, demonstration, training and testing phase, as well as registering test scores. The complete test protocol of the assessment is available online (Faber et al., 2016; https://doi.org/10.1371/journal.pone.0149037.s001 ). All children were tested under similar conditions as part of the event after they did a warm-up. Total testing time for each child was approximately $20 \mathrm{~min}$ for the perceptuo-motor tests spread over three sessions. Test leaders were physical therapy students or table tennis trainers who were familiarized with the use of the test protocol. Moreover, instruction and feedback were given during a practical training by an expert trainer of the NTTA.

\section{Questionnaire}

All participants were asked to fill in a questionnaire to obtain information about personal characteristics (sex (boy/girl), age (year), height $(\mathrm{cm})$, body mass $(\mathrm{kg})$, playing hand (left/ right)) and the foregoing sport-specific training. The quantity of their table tennis training was mapped by 1.) the current training (hours per week) and 2.) the total training volume (hours) based on average training hours per week of the seasons played. For the quality of the table tennis training, the qualification level of player's trainer was questioned. In the Netherlands there are five levels of trainer's qualifications: TT1 (youth guidance), TT2 (youth/assistant club trainer), TT3 (club trainer), TT4 (trainer of a regional training centre) and TT5 (national trainer). TT1 to TT3 are organized by the NTTA and the highest levels, TT4 and TT5, are offered by the Netherlands Olympic Committee*Netherlands Sports Federation (NOC*NSF) (www.nttb.nl). Additionally, a lack of coaching qualifications was taken into account. In consultation with the Dutch table tennis trainers association it was decided to use a binary outcome 
for the quality of training: high (i.e. TT4 and TT5) and low (i.e. no qualification, TT1, TT2 and TT3). High qualified trainers are professional part-time or full-time trainers at clubs or regional/national training centres. In general, these trainers are more experienced and obligated to participate in additional courses to keep their trainer's license. Low qualified trainers are mostly volunteers at clubs and often unpaid. The commitment to additional (refresher) courses are frequently waived by the clubs.

\section{Statistical analysis}

IBM SPSS Statistics 26 (IBM Corp., Armonk, New York, United States of America) was used for the statistical analyses. The normality of data was evaluated by comparing (1) means and medians of the test items and (2) standard deviation with minimal and maximal values. Sample characteristics and descriptive statistic were presented per sex. First, to determine the degree of association between the perceptuo-motor skills assessment outcomes and the quantity of the training non-parametric partial correlations were calculated per test item. Second, multivariate General Linear Model (GLM) analysis was used to test for differences regarding the test scores between youth players experiencing a high versus a low quality of training. Age was included as covariate as previous analyses showed that older youth players outperform younger players on the test items (Faber et al., 2014; Faber et al., 2016). Moreover, as boys tend to outscore girls and sex might have a modifying effect on the results, both the partial correlations and multivariate GLM analysis were conducted separately for boys and girls (Faber et al., 2014). Third, an additional multivariate GLM analysis was conducted and followed up with a discriminant analysis, both using age and sex normalised z-scores, to evaluate the relationship between both the quantity and quality of training experience and the perceptuo-motor skills assessment outcomes at once. For this purpose, four groups of youth players were defined based on their total training volume, and the trainer's qualification level; 1.) low volume and low quality, 2.) low volume and high quality, 3.) high volume and low quality and 4.) high volume and high quality. A low training volume was assigned when a player belonged to the $40 \%$ of the players with the lowest total training volume of his/her age group. A high training volume was assigned when the player belonged to the $40 \%$ players with the highest total training volume of his/her age group. The multivariate GLM analysis was used to test for differences between the four groups. The discriminant analysis, including the case wise results and "leave-one-out method" for crossvalidation, evaluated the perceptuo-motor assessment's capacity to classify the young table tennis players in the four groups. Cohen's rules of thumb are used for the interpretation of the effect sizes; partial correlation .10 small, .30 medium and .50 large and GLM's partial eta-squared .01 small, .06 medium and .14 large (Cohen, 1988). Alpha was set at .05 for significance for all analyses.

\section{Results}

In total, 372 young table tennis players (age 8-10 years) were assessed during the NTTA's national talent days (2010-2015). Table 2 presents the characteristics of the participants. The characteristics in girls and boys were similar, however more boys were tested compared to girls.

Table 3 shows the descriptive outcomes of the perceptuo-motor skills assessment and the questionnaire (i.e. sport-specific training information). All data collected with the perceptuomotor skills assessment were evaluated as normally distributed for both boys and girls; mean and medians were similar and the range around the mean followed a normal distribution. This was also the case for the subsamples used within further analyses. The training quantity outcomes (i.e. current training and training volume) on the other hand presented a right-skewed distribution of the data. 
Table 2.

Characteristics of participants

\begin{tabular}{|c|c|c|c|c|}
\hline & & Total & Boys & Girls \\
\hline \multirow[t]{7}{*}{ Total number } & $2010-2015$ & $372(100 \%)$ & $241(65 \%)$ & $131(35 \%)$ \\
\hline & 2010 & $68(18 \%)$ & 42 & 26 \\
\hline & 2011 & $72(19 \%)$ & 41 & 31 \\
\hline & 2012 & $92(25 \%)$ & 57 & 35 \\
\hline & 2013 & $50(13 \%)$ & 38 & 12 \\
\hline & 2014 & $36(10 \%)$ & 27 & 9 \\
\hline & 2015 & $54(15 \%)$ & 37 & 17 \\
\hline \multirow[t]{3}{*}{ Age group } & 8 years & $48(13 \%)$ & 29 & 19 \\
\hline & 9 years & $122(33 \%)$ & 82 & 40 \\
\hline & 10 years & $202(54 \%)$ & 131 & 71 \\
\hline \multirow[t]{2}{*}{ Handedness $(n=327)$} & left & $35(11 \%)$ & 26 & 9 \\
\hline & right & $292(89 \%)$ & 187 & 105 \\
\hline Standing height $(\mathrm{cm})$ & $\left(\mathrm{n}=323 ; \sigma^{\top} 211\right.$, ᄋ 112) & $142 \pm 8.1$ & $142 \pm 8.1$ & $142 \pm 8.2$ \\
\hline Body mass (kg) & $\left(\mathrm{n}=321 ; \sigma^{\top} 209\right.$, ㅇ 112) & $34 \pm 6.9$ & $34 \pm 6.5$ & $36 \pm 7.5$ \\
\hline BMI $\left(\mathrm{kg} / \mathrm{m}^{2}\right)$ & $(n=319 ;$ o' 207, ㅇ 112) & $16.9 \pm 2.4$ & $16.7 \pm 2.4$ & $17.4 \pm 2.5$ \\
\hline \multirow[t]{8}{*}{ Departments } & Holland-Noord & $37(10 \%)$ & 26 & 11 \\
\hline & Zuid-West & $37(10 \%)$ & 23 & 14 \\
\hline & West & $65(17 \%)$ & 46 & 19 \\
\hline & Noord & $28(8 \%)$ & 16 & 12 \\
\hline & Limburg & $55(15 \%)$ & 35 & 20 \\
\hline & Gelre & $40(11 \%)$ & 26 & 14 \\
\hline & Midden & $53(14 \%)$ & 35 & 18 \\
\hline & Oost & $57(15 \%)$ & 34 & 23 \\
\hline
\end{tabular}

Data are frequencies (and percentage of the total sample (\%)) except for standing height, weight and BMI. These latter variables are presented in means \pm standard deviations.

Table 3.

Outcomes perceptuo-motor skills assessment and training questionnaire

\begin{tabular}{lcccccc}
\hline & \multicolumn{2}{c}{ Total } & \multicolumn{2}{c}{ Boys } & \multicolumn{2}{c}{ Girls } \\
\hline Perceptuomotor skills assessment & $\mathrm{n}$ & $\mathrm{M} \pm \mathrm{SD}$ & $\mathrm{n}$ & $\mathrm{M} \pm \mathrm{SD}$ & $\mathrm{n}$ & $\mathrm{M} \pm \mathrm{SD}$ \\
\hline Sprint (s) & 369 & $34 \pm 4$ & 240 & $34 \pm 4$ & 129 & $35 \pm 3$ \\
Agility (s) & 367 & $30 \pm 8$ & 238 & $28 \pm 7$ & 129 & $33 \pm 9$ \\
Vertical jump (cm) & 368 & $29 \pm 6$ & 239 & $29 \pm 6$ & 129 & $28 \pm 5$ \\
Speed while dribbling (s) & 371 & $23 \pm 6$ & 241 & $22 \pm 5$ & 130 & $25 \pm 6$ \\
Aiming at target (points) & 371 & $24 \pm 10$ & 241 & $25 \pm 10$ & 130 & $22 \pm 10$ \\
Ball skills (points) & 368 & $17 \pm 6$ & 238 & $18 \pm 6$ & 130 & $15 \pm 6$ \\
Throwing a ball (m) & 369 & $10 \pm 2$ & 239 & $10 \pm 2$ & 130 & $9 \pm 2$ \\
Eye -hand coordination (points) & 369 & $15 \pm 7$ & 239 & $16 \pm 7$ & 130 & $13 \pm 7$
\end{tabular}




\begin{tabular}{lccccccc}
\hline Table 3 (continued) & \multicolumn{2}{c}{ Total } & Boys & & Girls \\
\hline Training quantity & $\mathrm{n}$ & Md (range) & $\mathrm{n}$ & Md (range) & $\mathrm{n}$ & Md (range) \\
\hline \multicolumn{1}{c}{ Current training (hours/week) } & 257 & $3(1-10)$ & 168 & $3(1-10)$ & 89 & $2.5(1-8.5)$ \\
& 8-year olds & 28 & $1.5(1-10)$ & 18 & $1.88(1-10)$ & 10 & $1(1-8)$ \\
& 9-year olds & 93 & $2.75(1-9.5)$ & 62 & $3(1-9.5)$ & 31 & $2.5(1-8.5)$ \\
& 10-year olds & 136 & $3(1-8)$ & 88 & $3(1-8)$ & 48 & $2.75(1-7)$ \\
Training volume (hours) & 257 & $140(4-1000)$ & 168 & $144(4-808)$ & 89 & $120(15-1000)$ \\
& 8-year olds & 28 & $70(25-408)$ & 18 & $72(25-408)$ & 10 & $64(28-408)$ \\
& 9-year olds & 93 & $120(15-808)$ & 62 & $137(24-808)$ & 31 & $110(15-548)$ \\
10-year olds & 136 & $148(4-1000)$ & 88 & $164(4-704)$ & 48 & $142(28-1000)$ \\
\hline Training quality & & $\mathrm{n}$ & percentage & $\mathrm{n}$ & percentage & $\mathrm{n}$ & percentage \\
\hline High & 71 & $27 \%$ & 43 & $26 \%$ & 28 & $31 \%$ \\
Low & 188 & $73 \%$ & 125 & $74 \%$ & 63 & $69 \%$ \\
\hline
\end{tabular}

$\mathrm{M}=$ mean; $\mathrm{SD}=$ standard deviation; $\mathrm{Md}=$ median.

Table 4.

Relationship between training and perceptuo-motor skills outcomes

\begin{tabular}{|c|c|c|c|c|c|c|c|}
\hline \multirow[b]{2}{*}{ Boys $(\mathrm{df}=163)$} & \multicolumn{2}{|c|}{$\begin{array}{l}\text { Current training } \\
\text { (hours/week) }\end{array}$} & \multicolumn{2}{|c|}{$\begin{array}{l}\text { Training volume } \\
\text { (hours) }\end{array}$} & \multicolumn{3}{|c|}{$\begin{array}{l}\text { Training quality (high versus } \\
\text { low) })^{1}\end{array}$} \\
\hline & $r$ & $p$ & $r$ & $p$ & $\mathrm{~F}^{2}$ & $p$ & partial $\eta^{2}$ \\
\hline Sprint (s) & -.022 & .778 & -.007 & .933 & .006 & .941 & $<.001$ \\
\hline Agility (s) & -.028 & .719 & .012 & .879 & .026 & .872 & $<.001$ \\
\hline Vertical jump (cm) & .025 & .746 & .142 & .070 & .744 & .390 & .005 \\
\hline Speed while dribbling (s) & $-.189 *$ & .015 & $-.230^{*}$ & .003 & $4.271^{*}$ & .040 & .026 \\
\hline Aiming at target (points) & $.345^{*}$ & $<.001$ & $.379 *$ & $<.001$ & .422 & .517 & .003 \\
\hline Ball skills (points) & -.003 & 0.967 & -.041 & .602 & .056 & .814 & $<.001$ \\
\hline Throwing a ball (m) & .126 & .108 & .063 & .421 & .027 & .869 & $<.001$ \\
\hline Eye-hand coordination (points) & $.214^{*}$ & .006 & $.250^{*}$ & .001 & 1.404 & .238 & .009 \\
\hline Girls $(\mathrm{df}=84)$ & $r$ & $p$ & $r$ & $p$ & $\mathrm{~F}^{2}$ & $p$ & partial $\eta^{2}$ \\
\hline Sprint (s) & -.134 & .220 & .043 & .695 & 1.971 & .164 & .022 \\
\hline Agility (s) & -.125 & .250 & -.118 & .278 & .135 & .714 & .002 \\
\hline Vertical jump (cm) & .058 & .595 & .058 & .595 & .357 & .552 & .004 \\
\hline Speed while dribbling (s) & $-.405^{*}$ & $<.001$ & $-.365^{*}$ & .001 & .554 & .459 & .006 \\
\hline Aiming at target (points) & $.352 *$ & .001 & $.396^{*}$ & $<.001$ & 2.045 & .156 & .023 \\
\hline Ball skills (points) & .063 & .561 & .149 & .172 & .001 & .974 & $<.001$ \\
\hline Throwing a ball (m) & .148 & .174 & .137 & .208 & .027 & .870 & $<.001$ \\
\hline Eye -hand coordination (points) & $.264^{*}$ & .014 & $.224^{*}$ & .039 & .119 & .731 & .001 \\
\hline
\end{tabular}


The non-parametric partial correlation analyses showed that the current training (hours per week) and three perceptuo-motor test items were significantly associated with small to medium effect sizes in both boys and girls (Table 4); speed while dribbling (ơ $: r=-.189, p=.015 ;$ ㅇ $: r=-.405, p<$ $.001)$, aiming at target $\left(\sigma^{7}: r=.345, p<.001 ; \%: r\right.$ $=.352, p=.001)$, eye-hand coordination ( $\sigma^{7}: r=$ $.214, p=.006$; $q: r=.264, p=.014)$. A similar tendency was seen for the association between the training volume (hours) and the perceptuo-motor test items in both sexes; speed while dribbling speed while dribbling ( $\sigma^{*}: r=-.230, p=.003 ; \%: r=-.365$, $p=.001)$, aiming at target $\left(\sigma^{\prime}: r=.379, p<.001\right.$; q: $r=.396, p<.001)$, eye-hand coordination ( $\sigma^{\prime}: r$ $=.250, p=.001 ; \%: r=.224, p=.039)$. No other significant associations were found.

The multivariate GLM analysis revealed no significant differences between players that received training from a high quality trainers versus players that received training from a low quality trainer in boys $(\Lambda=.965, \mathrm{~F}(8,156)=.707, p=.685)$ and also in girls $(\Lambda=.928, \mathrm{~F}(8,79)=.771, p=.629)$. The univariate GLM results are summarized in Table 4, revealing one significant difference for speed while dribbling in which the male youth players receiving high quality training outscored their peers receiving a low-quality training $(\mathrm{F}=4.271, \mathrm{p}=.040)$.
Nevertheless, the effect size was small (partial $\eta^{2}=$ .026).

The multivariate GLM analysis testing difference between the four groups based on training volume and trainer's qualification level showed significant effect regarding the perceptuo-motor assessment outcomes $(\Lambda=.728, \mathrm{~F}(24,549)=2.647, p<.001)$. Significant difference with medium to large effect sizes between the groups were present at the test items: speed while dribbling $(\mathrm{F}(3)=7.331, p<.001$, partial $\left.\eta^{2}=.101\right)$, aiming at target $(\mathrm{F}(3)=13.150, p$ $<.001$, partial $\left.\eta^{2}=.168\right)$, throwing a ball $(\mathrm{F}(3)=$ 3.744, $p=.012$, partial $\left.\eta^{2}=.054\right)$, and eye hand coordination $\left(\mathrm{F}(3)=5.422, p<.001\right.$, partial $\eta^{2}=$ .077). The consecutive discriminant analysis indicated three discriminant functions $\mathrm{F} 1 \quad \mathrm{r}_{\mathrm{can}}=$ .481 ; Wilks $\lambda=.728 ; p<.001)$, F2 ( $\mathrm{r}_{\mathrm{can}}=.205$; Wilks $\lambda=.947 ; p=.721)$ and $\mathrm{F} 3\left(\mathrm{r}_{\mathrm{can}}=.108\right.$; Wilks $\lambda=$ $.988 ; p=.892$ ) accounting for $84 \%, 13 \%$ and $6 \%$ of the variance, respectively. On the whole, $51 \%$ of the cross-validated grouped cases were correctly classified into the four groups defined by training volume and trainer's quality based on the assessment outcomes (Table 5). The correct classifications were only noticeable in group 1 (low volume and low quality) and group 3 (high volume and low quality). None of the youth players from the groups with a high-qualified trainer (i.e. group 2 and 4) were qualified correctly.

Table 5.

Discriminant analysis cross-validated classification results

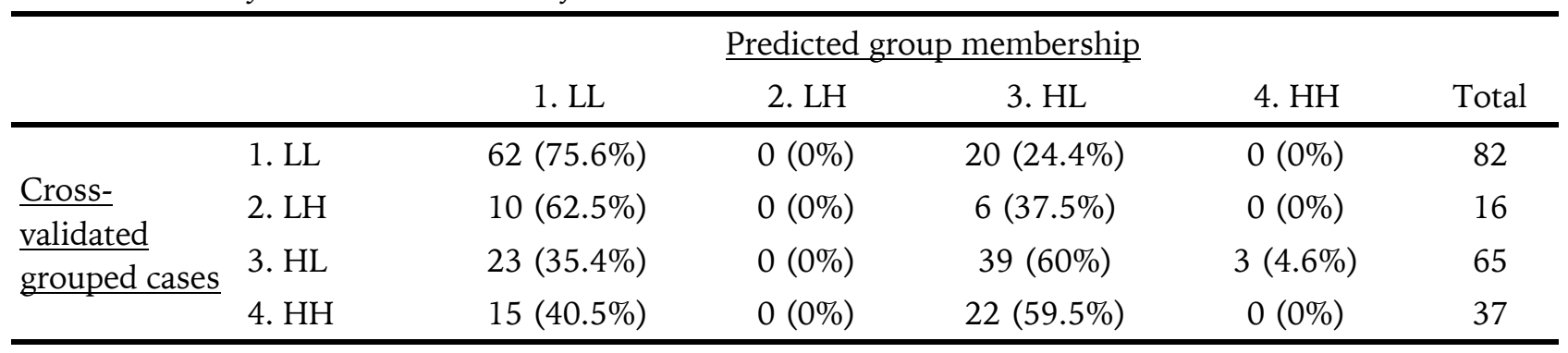

Data are number and percentages. Groups: 1.) low volume and low quality, 2.) low volume and high quality, 3.) high volume and low quality and 4.) high volume and high quality. The analysis indicated three discriminant functions F1 ( $\mathrm{r}_{\mathrm{can}}=.481$; Wilks $\left.\lambda=.728 ; p<.001\right)$, F2 $\left(\mathrm{r}_{\mathrm{can}}=0.205\right.$; Wilks $\lambda=.947 ; p$ $=.721)$ and $\mathrm{F} 3\left(\mathrm{r}_{\mathrm{can}}=.108\right.$; Wilks $\left.\lambda=.988 ; p=.892\right)$ accounting for $84 \%, 13 \%$ and $6 \%$ of the variance, respectively. 


\section{Discussion}

This study focused on the relationship between the sport-specific training and the outcomes of the perceptuo-motor skills assessment in youth table tennis players (8-12 years) used as a part of talent identification. The results of this study show significant relationships with small to medium effect sizes between three of the included test items (i.e. speed while dribbling, aiming at target and eye hand coordination) and the training quantity outcomes. This means that a part of the test outcomes is indeed associated to a certain extent with the sport-specific training. This might imply that training quantity should be taken into account when interpreting the test outcomes of these test items for the estimation of potential. Furthermore, the relation between the quality of training and the test outcomes seems to be less important or even negligible based on the results of the multivariate GLM analyses and the consecutive discriminant analysis.

The significant relationship between the quantity of the table tennis training and the three test items on the one hand and the lack of association between training quantity and the other test items on the other hand, might be best explained by the differentiation in task characteristics. Speed while dribbling, aiming at target and eye hand coordination all belong to the construct 'ball control' (Faber et al., 2015). These items cover object manipulation while controlling a ball, probably by deploying the visuomotor system for visual acuity and eye-hand coordination in different circumstances. These characteristics are more similar to the exercises conducted during the sport-specific table tennis training and the actual table tennis play compared to the task characteristics of the other test items. Speed while dribbling and eye hand coordination mimic the rhythm and the back and forth going of the ball and the aiming tasks includes the use of a table tennis bat in a hitting task. As such, transfer of skill is more likely to occur in these tasks compared to the others (Côté et al., 2007; Schmidt \& Lee, 2011). Although , the test items 'ball skills' and 'throwing a ball' also belong to the construct 'ball control', these items' characteristics seem less specific, since youth players are requested to throw a ball. The other test items (i.e. sprint, agility and vertical jump) form the construct 'gross motor function' combining strength, speed agility, which are also less specific tasks. Hence, the characteristics of speed while dribbling, aiming at target and eye hand coordination appear to be more near transfer tasks in comparison with the other test items. Near and far transfer are considered according to the degree of similarity between two tasks, assuming that near transfer tasks exist when the task constraints and performance information are quite similar to the initial settings (Issurin, 2013). Nevertheless, the effect sizes are low to moderate, which indicates a low degree of skill transfer and certain degree of task specificity (de Camargo Barros et al., 2017; Ellison et al., 2018).

It is important to acknowledge some limitations of this study. First, it must be mentioned that this study used a cross-sectional design, which prohibits any conclusions about causality. A study with a longitudinal experimental design is needed to provide a fair conclusion about the effect of sport specific table tennis training on the outcomes of the perceptuo-motor skills assessment. Second, this study learned that it was challenging to estimate the quality of the sport specific training based on the questionnaire used. Two reasons for this seem to be most legitimate. To start with, it can be argued whether the qualification of the trainer alone reflects the trainer's competencies in the best way. For example, no attention was paid to the years of experience, the experience with youth athletes, the successes achieved and the participation in refreshing course. Moreover, many players appeared to have more trainers. The highest qualified trainer was taken into account for study, which might not be the 'leading' trainer or the trainer that the youth players mainly receive their training from. These two factors might have influenced the results. However, it is unclear in what direction. Third, no information about the type of training, the variability within the training, training experience in other sports, physical education programs or other physical activity (structured or unstructured) have been taken into 
account in this study. This might have influenced the study results (Seifert, Papet, Strafford, Gogliani, \& Davids, 2019), but it is unknown to what extent it should be taken into account or be corrected for when estimating the potential of young table tennis players. Finally, it must be mentioned that we missed results on training experience from approximately $30 \%$ of the included sample, since not all participants handed in the questionnaire. Only the available data was taken into account for the analysis. Although a substantial part of the sample could be included, there may a response-bias.

\section{Conclusions}

In conclusion, there appears to be a significant relationship between the sport specific training and three test items of the perceptuo-motor skills assessment in youth table tennis players (8-10 years) participating at the national talent day. The relationship is determined by the training quantity. The quality of the training seems of less influence. The test items showing a significant association with the assessment test outcomes are considered to be more similar to the table tennis training exercises when compared to the other test items. Still, the effect sizes of the association are small to moderate, which suggests only a small transfer and still a substantial amount of task specificity (de Camargo Barros et al., 2017; Issurin, 2013). For that reason, it seems legitimate to continue to use the perceptuomotor skills assessment as part of the talent identification in youth table tennis. However, for a fair interpretation of the estimation of potential, it seems sensible to take the training volume into account, especially when large differences exist between players regarding the training experience. Future studies should reveal more about the effect of training in a longitudinal design while improving the determination of the training quality.

\section{Acknowledgments}

We acknowledge the Netherlands Table Tennis Association for the provision of the data, the trainers of the Netherlands Table Tennis Association and the physiotherapy students of Saxion University of Applied Sciences for their help with the assessments and, of course, all of the children and parents for their participation in this study. Special thanks go to Achim Sialino, technical director of the Netherlands Table Tennis Association, who supported this research from the start.

\section{Funding \& Conflict of interests}

No funding was provided for the conductance of this study. The authors declare no conflict of interests.

\section{References}

Behringer, M., vom Heede, A., Matthews, M., \& Mester, J. (2011). Effects of strength training on motor performance skills in children and adolescents: a meta-analysis. Pediatric exercise science, 23(2), 186-206.

Cohen, J. (1988). Statistical power analysis for the behavioral sciences. Abingdon: Routledge.

Collins, R., Collins, D., MacNamara, Á., \& Jones, M. I. (2014). Change of plans: an evaluation of the effectiveness and underlying mechanisms of successful talent transfer. Journal of sports sciences, 32 (17), 1621-1630.

Côté, J., Baker, J., \& Abernethy, B. (2007). Practice and play in the development of sport expertise. In: R. Eklund \& G. Tenenbaum (Eds.), Handbook of sport psychology (3 edition). Hoboken, NJ; Wiley.

de Bosscher, V., Bingham, J., Shibli, S., Bottenburg, M. van, \& Knop, P. de (2007). The global sporting arms race. An international comparative study on sports policy factors leading to international sporting success. Oxford: Meyer \& Meyer Sport.

de Camargo Barros, J. A., Tani, G., \& Corrêa, U. C. (2017). Effects of practice schedule and task specificity on the adaptive process of motor learning. Human movement science, 55, 196-210.

Elferink-Gemser, M. T. (2005). Today's talented youth field hockey players, the stars of tomorrow?: a study (Doctoral dissertation). Groningen: ElferinkGemser. 
Elferink-Gemser, M., Jordet, G., Coelho E Silva, M. J., \& Visscher, C. (2011). The marvels of elite sports: How to get there? British Journal of Sports Medicine, 45, 683-684.

Ellison, P. H., Kearney, P. E., Sparks, S. A., Murphy, P. N., \& Marchant, D. C. (2018). Further evidence against eye-hand coordination as a general ability. International Journal of Sports Science \& Coaching, 13(5), 687-693.

Ericsson, K. A., Krampe, R. T. \& Tesch-Romer, C. (1993). The role of deliberate practice in the acquisition of expert performance. Psychological Review, 100, 363-406.

Faber, I. R., Elferink-Gemser, M. T., Oosterveld, F. G. J., \& Nijhuis-Van Der Sanden, M. W. G. (2014a). Revision of two test items of the Dutch motor skills assessment measuring ball control in young table tennis players: A reproducibility and validity study. Annals of Research in Sport and Physical Activity, 5, 5369.

Faber, I. R., Oosterveld, F. G. \& Nijhuis-Van der Sanden, M. W. (2014b). Does an eye-hand coordination test have added value as part of talent identification in table tennis? A validity and reproducibility study. PloS one, 9(1), e85657.

Faber, I. R., Nijhuis-Van Der Sanden, M. W., Elferink-Gemser, M. T., \& Oosterveld, F. G. (2015). The Dutch motor skills assessment as tool for talent development in table tennis: a reproducibility and validity study. J Sport Sci, 33(11), 1149-1158.

Faber, I. R. (2016). Diamonds in the rough. Searching for high potential in youth table tennis players (Doctoral dissertation). Twello: Faber.

Faber, I. R., Elferink-Gemser, M. T., Faber, N. R., Oosterveld, F. G., \& Nijhuis-Van der Sanden, M. W. (2016). Can perceptuo-motor skills assessment outcomes in young table tennis players (7-11 years) predict future competition participation and performance? An observational prospective study. PloS one, 11 (2), e0149037.

Faber, I. R., Pion, J., Munivrana, G., Faber, N. R., \& Nijhuis-Van der Sanden, M. W. (2018). Does a perceptuomotor skills assessment have added value to detect talent for table tennis in primary school children? Journal of sports sciences, 36(23), 2716-2723.
Faber, I. R., Damsma, T., \& Pion, J. (in press). Chapter 8: Finding talent and establishing the road to excellence in table tennis - The Dutch case. In: J. Baker, S. Cobley, \& J. Schorer (Eds.), Talent Identification and Development in Sport: International Perspectives (2 ${ }^{\text {nd }}$ edition). Abingdon: Routledge.

Farhat, F., Hsairi, I., Baati, H., Smits-Engelsman, B. C. M., Masmoudi, K., Mchirgui, R., Triki, C., \& Moalla, W. (2016). The effect of a motor skills training program in the improvement of practiced and non-practiced tasks performance in children with developmental coordination disorder (DCD). Human movement science, 46, 10-22.

Gagné, F. (2004). Transforming gifts into talents: The DMGT as a developmental theory. High ability studies, 15(2), 119-147.

Gamble, P. (2006). Implications and applications of training specificity for coaches and athletes. Strength Cond J, 28(3), 54-58.

Huijgen, B. C. H. (2013). Technical skills, the key to succes? A study on talent development and selection of youth soccer players (Doctoral dissertation). Groningen: Huijgen.

Issurin, V. B. (2013). Training transfer: scientific background and insights for practical application. Sports Med, 43, 675-694.

Kramer, T. (2019). How to develop a Grand Slam winner... Physical and psychological skills in Dutch junior tennis players (Doctoral dissertation). Groningen: Kramer.

Morrow, J. R., Jackson, A. W., Disch, J. G., \& Mood, D. P. (2011). Measurement and evaluation in human performance (4 ${ }^{\text {th }}$ ed.). Champaign IL: Human Kinetics.

Pion, J. (2015). The Flemish sports compass: From sports orientation to elite performance prediction (Doctoral dissertation). Ghent: Ghent University.

Pion, J., Hohmann, A., Liu, T., Lenoir, M., \& Segers, V. (2017). Predictive models reduce talent development costs in female gymnastics. Journal of sports sciences, 35(8), 806-811.

Platvoet, S., Faber, I. R., De Niet, M., Kannekens, R., Pion, J., Elferink-Gemser, M. T., \& Visscher, C. (2018, September). Development of a tool to assess fundamental movement skills in applied settings. 
Frontiers in Education, 3:75. doi: 10.3389/feduc.2018.00075.

Rea, T., \& Lavallee, D. (2015). An examination of athletes' experiences of the talent transfer process. Talent Development and Excellence, 7(1), 41-67.

Rylander, P., Karlsteen, M., Kougioumtzis, K., \& Gustafsson, J. E. (2019). The specificity versus generality of ball-handling skills-Empirical evidence for a general ball-handling ability. Human movement science, 66, 477-486.

Schmidt, R. A., \& Lee, T. D. (2011). Motor control and learning: a behavioral emphasis ( $5^{\text {th }}$ ed.). Champaign, IL: Human Kinetics.

Seifert, L., Papet, V., Strafford, B. W., Gogliani, A., \& Davids, K. (2019). Skill transfer, expertise and talent development: An ecological dynamics perspective. Movement \& Sport Sciences, 102(4), 39-49.
Sigmundsson, H., Trana, L., Polman, R., \& Haga, M. (2017). What is trained develops! theoretical perspective on skill learning. Sports, 5(2), 38.

Stoter, I. K. (2020). Staying on track - The road to elite performance in $1500 m$ speed skating (Doctoral dissertation). Groningen: Stoter

Thorndike, E. L. (1914). Education psychology: Briefer course. Abingdon: Routledge.

Vaeyens, R., Lenoir, M., Williams, A. M., \& Philippaerts, R. M. (2008). Talent identification and development programmes in sport. Sports medicine, 38(9), 703-714.

Vandorpe, B., Vandendriessche, J. B., Vaeyens, R., Pion, J., Lefevre, J., Philippaerts, R. M., \& Lenoir, M. (2012). The value of a non-sport-specific motor test battery in predicting performance in young female gymnasts. Journal of Sports Sciences, 30(5), 497-505. 